\title{
Pre-irradiation of mouse mammary gland stimulates cancer cell migration and development of lung metastases
}

\author{
G Bouchard ${ }^{1}$, G Bouvette ${ }^{1}$, H Therriault ${ }^{1}$, R Bujold ${ }^{1,2}$, C Saucier ${ }^{3}$ and B Paquette ${ }^{*, 1}$ \\ ${ }^{1}$ Centre for Research in Radiotherapy, Department of Nuclear Medicine and Radiobiology, Université de Sherbrooke, 3001, \\ 12e Avenue Nord, Sherbrooke, Québec J1H 5N4, Canada; ${ }^{2}$ Service of Radiation Oncology, Centre Hospitalier Universitaire \\ de Sherbrooke, Sherbrooke, Québec, Canada and ${ }^{3}$ Department of Anatomy and Cellular Biology, Faculty of Medicine and Health \\ Sciences, Université de Sherbrooke, Sherbrooke, Québec, Canada
}

Background: In most patients with breast cancer, radiotherapy induces inflammation that is characterised by an increase of promigratory factors in healthy tissues surrounding the tumour. However, their role in the emergence of the migration phenotype and formation of metastases is still unclear.

Methods: A single mammary gland of BALB/c mice was irradiated with four doses of 6 Gy given at a 24-h interval. After the last session of irradiation, treated and control mammary glands were either collected for quantification of promigratory and proinflammatory factors or were implanted with fluorescent ubiquitination-based cell cycle indicator (FUCCl)-expressing mouse mammary cancer D2A1 cells. The migration of cancer cells in the mammary glands was monitored by optical imaging. On day 21, mammary tumours and lungs were collected for histology analyses and the quantification of metastases.

Results: Pre-irradiation of the mammary gland increased by 1.8-fold the migration of cancer cells, by 2-fold the quantity of circulating cancer cells and by 2.4-fold the number of lung metastases. These adverse effects were associated with the induction of interleukin-6 (IL-6) and cyclooxygenase-2 (COX-2).

Conclusion: The emergence of the metastasis phenotype is believed to be associated with the accumulation of mutations in cancer cells. Our results suggest an alternative mechanism based on promigratory factors from irradiated mammary glands. In clinic, the efficiency of radiotherapy could be improved by anti-inflammatory agents that would prevent the stimulation of cancer cell migration induced by radiation.

Radiotherapy is an important part of breast cancer treatment. This modality can completely cure the disease or eliminate a large number of cancer cells, and it can reduce the recurrence rate and increase the overall survival of patients. It is worth noting that the total radiation dose is limited by the tolerance of surrounding normal tissues and is not meant to eradicate all cancer cells scattered in the breast, but rather to optimise long-term results with minimal adverse effects. Consequently, women still have a nonnegligible risk of breast cancer death after radiotherapy (Clarke et al, 2005). Clinicians strive to increase the effectiveness of radiotherapy within acceptable limits of host toxicity, which aim to minimise adverse effects such as inflammation of normal tissues potentially causing fibrosis or dermatitis.

Radiotherapy is recognised to trigger an inflammatory response (Gallet et al, 2011). This inflammation is characterised by an increase of cytokines, angiogenic factors, adhesion molecules and matrix metalloproteinases (MMPs) (Rodemann and Blaese, 2007). It is also known that chronic inflammation increases the risk of developing several types of cancer, including breast cancer (Mantovani et al, 2008). Observations also suggest that radiation 
might promote the invasiveness of breast cancer cells (Madani et al, 2008). For instance, we recently reported that mouse thighs that were pre-irradiated increased the invasiveness of implanted mammary cancer cells (Lemay et al, 2011). Another study demonstrated that radiation promoted changes in the mammary gland stromal microenvironment that contributed to the tumourigenic potential of breast cancer cells (Barcellos-Hoff and Ravani, 2000). However, the functional roles of the promigratory molecules induced by radiation during the early phase of the metastatic cascade remain unresolved. A better understanding of the alleged prometastatic properties of radiation could contribute to the development of new therapeutic modalities to prevent these undesirable effects.

The evidence linking the microenvironment to tumour progression is growing (Goldberg and Schwertfeger, 2010). In an effort to determine the role of irradiation in the progression of breast cancer, we pre-irradiated a mouse mammary gland and then implanted triple-negative mammary carcinoma cells D2A1. This protocol allowed us to specifically assess whether inflammation induced by radiation could stimulate the progression of cancer cells. Our procedure had the advantage of defining the mechanisms involved and eliminating confounding effects that could occur by irradiating the tumour and the mammary gland at the same time. Some examples of confounding effects are the selection of cancer cells more likely to migrate or the induction of mutations that would increase the aggressiveness of tumour cells.

Although the mammary gland radiation-induced stromal effect and carcinogenesis have been studied (Barcellos-Hoff, 2010), we are, to our knowledge, the first to investigate a preclinical model of breast cancer recurrence following standard fractionated radiotherapy. Our innovative mouse model of triple-negative breast cancer cell migration is a step forward in the understanding of metastatic breast cancer.

In this study, mouse mammary glands were pre-irradiated that stimulated the migration of mammary cancer cells at the primary site of implantation, increased the number of circulating cancer cells and promoted lung metastases. These adverse effects of radiation were associated with the increased expression in the irradiated tissue of the key pro-inflammatory factors, cyclooxygenase-2 (COX-2) and interleukin-6 (IL-6).

\section{MATERIALS AND METHODS}

Cell culture. The mouse D2A1 cancer cells, kindly provided by Dr Ann F Chambers (University of Western Ontario, London, ON, Canada), are derived from a spontaneous mammary tumour in a $\mathrm{BALB} / \mathrm{c}$ mouse (Rak et al, 1992). These cells were maintained in a $5 \% \mathrm{CO}_{2}$ humidified incubator at $37^{\circ} \mathrm{C}$ in modified Eagle's medium (MEM) (Sigma-Aldrich, Oakville, ON, Canada) supplemented with $10 \%$ fetal bovine serum (Wisent, St Bruno, QC, Canada), $2 \mathrm{~mm}$ glutamine, $1 \mathrm{~mm}$ sodium pyruvate, 100 units per $\mathrm{ml}$ penicillin and $100 \mu \mathrm{M}$ streptomycin.

Migration capacity of $\mathrm{D} 2 \mathrm{~A} 1$ cells assessed in invasion chambers. For the invasion assay, BALB/c 3T3 fibroblasts $\left(2.5 \times 10^{4}\right)$ were seeded with MEM supplemented with $10 \%$ FBS in 24-well plates. After $20 \mathrm{~h}$, the cell culture medium was replaced with MEM supplemented with $0.1 \%$ bovine serum albumin (BSA) following two rinses in PBS. Cells were then irradiated using a ${ }^{60} \mathrm{Co}$ source (Gammacell 220, Nordion, Canada) at a dose of $5 \mathrm{~Gy}$. Shamirradiated cells were used as a control. The fibroblast conditioned media were used as a chemoattractant in the lower compartment of the invasion chambers (Becton Dickinson Biosciences, Bedford, MA, USA). Invasion chambers coated with Matrigel (artificial basement membrane) were rehydrated with $1 \mathrm{ml}$ MEM $0.1 \%$ BSA for $2 \mathrm{~h}$ at $37^{\circ} \mathrm{C}$. Nonirradiated D2A1 mouse mammary cancer cells harvested with Cell Dissociation Solution (Sigma-Aldrich) were added $\left(4 \times 10^{4}\right)$ to the upper compartment of the invasion chambers $24 \mathrm{~h}$ after irradiation of the BALB/c 3T3 cells. Mouse mammary cancer cells that had passed across the Matrigel and the porous membrane $24 \mathrm{~h}$ later were fixed, stained with crystal violet and counted under the microscope. Results were reported as radiation-enhancement ratio. Each experiment was performed in triplicate and repeated three times.

Generation of D2A1 cells expressing the fluorescent ubiquitination-based cell cycle indicator (FUCCI) proteins. Genes encoding for the FUCCI proteins were introduced into the D2A1 cells to allow the detection and assessment of their cell cycle state by optical imaging. Replication-defective, self-inactivating CSII-EF-MCS lentiviral vectors encoding for Cdt1 and the Geminin E3 ligases substrates fused, respectively, to the red monomeric version of the Kusabira Orange (mKO2-hCdt1) and the green monomeric Azami Green (mAG-hGem) fluorescent proteins, which were generously provided by Dr Asako SakaueSawano (Brain Science Institute, RIKEN, Wako, Saitama, Japan). Red and green fluorescence are respectively markers of cells within the G1 (red) and S/G2/M (green) phases of the cell cycle (SakaueSawano et al, 2008). Each construct was co-transfected with plasmids encoding for the lentiviral packaging proteins (plp1, plp2 and plp/VSVG) in human embryonic kidney 293T cells using Lipofectamine 2000 according to the manufacturer's instructions (Invitrogen, Burlington, ON, Canada). After a 48-h incubation, lentivirus-containing supernatants were harvested and filtered with a $0.45-\mu \mathrm{m}$ filter, and then kept at $-80^{\circ} \mathrm{C}$ until further use. D2A1 cell population expressing the FUCCI proteins were generated following a triple sequential infection for each fluorescent protein (Wu et al, 2010).

Mammary gland pre-irradiation and injection of D2A1 FUCCIexpressing cells. The experimental protocols were approved by the institutional ethics committee and conformed to the regulations of the Canadian Council on Animal Care. Female retired breeder BALB/c mice (18-24 weeks old) were obtained from Charles River (Raleigh, NC, USA). Animals were anaesthetised with $3 \%$ isoflurane and then immobilised with a stereotactic mouse frame adapted to dock on the Leskell Gamma Knife Perfexion (Elekta, Stockholm, Sweden). The third right mammary gland was irradiated by an energy deposition of elliptical shape (Figure 1A). Anaesthetised mice were irradiated at a dose rate of $1.33 \mathrm{~Gy} \mathrm{~min}^{-1}$ to a total of $6 \mathrm{~Gy}$ during each of the 4 fractions at $24 \mathrm{~h}$ intervals. Based on dosimetry performed by our institutional medical physicist team, this protocol provided a biological effective dose (BED) comparable to the standard clinical regimen of $20 \times 2.25 \mathrm{~Gy}$, without having to perform daily anaesthesia over 20 days that would be lethal in mice. Regarding the nonirradiated mammary glands, they received a residual dose of $<1 \%$. To determine whether pre-irradiation of the mammary gland stimulated the migration of mouse mammary cancer cells, D2A1 FUCCI-expressing cells $\left(10^{6}\right.$ per $100 \mu \mathrm{l}$ PBS) were injected $3 \mathrm{~h}$ after the last irradiation into the pre-irradiated (right side) and unirradiated (control, left side) mammary glands. Mouse mammary carcinoma cells were also implanted into the mammary glands of sham-irradiated mice. The tumour volume was measured every 3 days by external caliper measurements and calculated with the formula: $V\left(\mathrm{~mm}^{3}\right)=\pi / 6 \times a(\mathrm{~mm}) \times b^{2}\left(\mathrm{~mm}^{2}\right)$, where ' $a$ ' and ' $b$ ' are the largest and smallest perpendicular tumour diameters, respectively (Balin-Gauthier et al, 2006). In other experiments, the D2A1 FUCCI-expressing cells $\left(10^{6}\right.$ per $100 \mu \mathrm{l}$ PBS) were instead injected intravenously via the tail vein of sham $(n=4)$ and preirradiated mice $(n=4)$. After 9 days, these animals were killed and their lungs were processed to quantify the number of metastases. The number and the diameter of lung metastases were quantified using the CellProfiler 2.0.0 software. Parameters were set to an intensity-based identification method on images containing a 


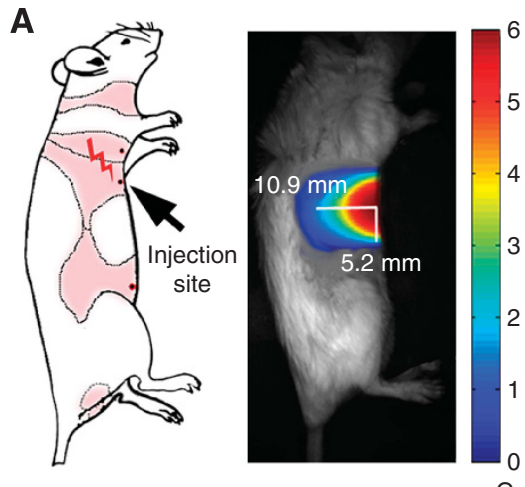

B

4x6 Gy irradiation of the mammary gland

$$
\begin{gathered}
\text { (24 h interval) } \quad \text { In vivo optical } \\
\text { imaging (week 1) }
\end{gathered}
$$

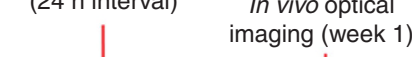

Animals killed, tissue sampling

and lungs imaging

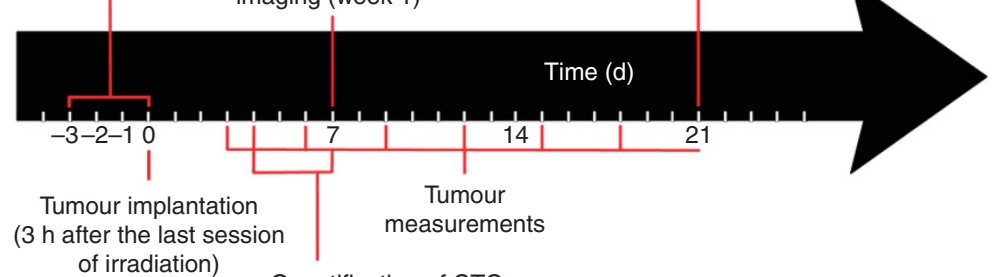

( $3 \mathrm{~h}$ after the last session

of irradiation)

Gy

Quantification of CTC

Figure 1. Description of in vivo irradiation model and study design. (A) Mouse model of irradiation, dosimetry and implantation of D2A1 cancer cells (figure adapted from EL Green, Editor, Dover publications, 1966). (B) Study design for the effects of radiation on mouse mammary cancer cell migration in pre-irradiated mice mammary glands.

dense amount of cancerous growth or a shape-based identification method on images containing a sparse amount of metastasis, using the fixed parameters. Figure $1 \mathrm{~B}$ summarises the chronological order of irradiation and the handling of animals.

In vivo and in situ optical imaging. The migration of D2A1 FUCCI-expressing cancer cells in the mammary gland was monitored with an animal optical imager (QOS Imager, Quidd S.A.S., Val de Reuil, France). Mice were anaesthetised with ketamine/xylazine $\left(87: 13 \mathrm{mg} \mathrm{ml}^{-1}\right.$ at $\left.1 \mathrm{mg} \mathrm{kg}^{-1}\right)$. A bright field image of the mice was taken and then the appropriate filters were selected for red and green fluorescent image acquisition $(\mathrm{mKO} 2$, $\lambda \mathrm{ex}=472 / 30, \lambda \mathrm{em}=536 / 40 ; \mathrm{mAG}, \lambda \mathrm{ex}=531 / 40, \lambda \mathrm{em}=593 / 40)$. The three images acquired were merged for future analysis. Distances of D2A1 cell migration in irradiated and nonirradiated mammary glands were measured to determine the radiationenhancement ratio. Migration was quantified with ImageJ (NIH, USA) as the distance from the nipple (physical landmark for injection site) to the end of fluorescent smear. On day 21, mice were killed, and tumour and lung specimens were removed (sham; $n=12$ sham, irradiated; $n=9$ ). Fluorescence images of the lungs were acquired and lungs metastases were quantified as described above.

Histology. Mammary tumours and lung specimens containing D2A1 FUCCI-expressing cancer cells were collected and immediately frozen in a solution of Optimum Cutting Temperature (OCT; Electron Microscopy Sciences, Hatfield, PA, USA). Cryosections of $3 \mu \mathrm{m}$ were cut using a Leica CM3050 Microsystems cryostat (GmbH, Wetzlar, Germany). Slides were dried for $30 \mathrm{~min}$ at $37^{\circ} \mathrm{C}$ and then stored at $-80^{\circ} \mathrm{C}$ until further use. The fluorescence emitted by the D2A1 cells was recorded using the FSX100 Bio Imaging Navigator microscope (Olympus, Center Valley, PA, USA) equipped with band pass filters (Chroma Technology Corp., Bellows Falls, VT, USA) for fluorescein isothiocyanate (FITC; $\lambda \mathrm{ex}=480 / 30, \lambda \mathrm{em}=535 / 40)$ or tetramethylrhodamine isothiocyanate (TRITC; $\lambda \mathrm{ex}=560 / 40, \lambda \mathrm{em}=630 / 60$ ). To calculate the ratio of red-to-green fluorescence intensity of cells in the tumours, the entire slide was scanned (magnification $\times 42$ ) and every image was quantified for red and green signals.

Quantification of inflammatory and pro-migratory factors. In different groups of irradiated mice ( $n=6$ per group), animals were killed at 4,7 or $24 \mathrm{~h}$ post irradiation, and their mammary glands were removed and snap frozen. Prostaglandin D2 $\left(\mathrm{PGD}_{2}\right)$ and E2 $\left(\mathrm{PGE}_{2}\right)$ levels were quantified by liquid chromatography/tandem mass spectrometry (LC-MS/MS) (Yang et al, 2002). The mRNA levels of COX-2, 15-hydroxyprostaglandin dehydrogenase (15-PGDH), IL-1 $\beta$, IL-6, membrane type 1 metalloprotease
(MT1-MMP), phospholipase A2 (PLA2), transforming growth factor- $\beta 1$ (TGF- $\beta 1$ ) and tumour necrosis factor- $\alpha$ (TNF- $\alpha$ ) were determined by quantitative real-time PCR(qPCR) in irradiated and contralateral nonirradiated mammary glands $6 \mathrm{~h}$ after the last session of irradiation $(n=6)$. Tissues were submerged in RNAlaterTM (Qiagen Inc., Toronto, ON, Canada) stored at $4{ }^{\circ} \mathrm{C}$ for $24 \mathrm{~h}$ and then at $-80^{\circ} \mathrm{C}$. Total RNA extractions, reverse transcription, primer dilutions and PCR reactions were made with the FastStart Universal SYBR Green Master mix (Roche Diagnostics, QC, Canada). The following cycling conditions were used: $10 \mathrm{~min}$ at $95^{\circ} \mathrm{C}$, and then 50 cycles of $15 \mathrm{~s}$ at $95^{\circ} \mathrm{C}, 30 \mathrm{~s}$ at $60^{\circ} \mathrm{C}$ and $30 \mathrm{~s}$ at $72^{\circ} \mathrm{C}$. Relative expression levels were calculated using the qBASE framework and normalised to the mouse UBC, HPRT1 and GAPDH housekeeping genes (Desmarais et al, 2012). The sequences of the primers that were used are listed in Supplementary Table 1 in the Supplementary Materials. MMP-2 and MMP-9 levels were analysed by zymography in mammary glands and tumour tissues in either irradiated or sham animals, using methods previously described $(n=6)$ (Lemay et al, 2011) and were also confirmed by immunohistochemistry (IHC) on $3 \mu \mathrm{m}$ paraffin-embedded tissues. The signal revelation of MMP-2 (Thermo Scientific, IL, USA) and MMP-9 (Antibodies-Online Inc., GA, USA) antibodies was realised using an anti-rabbit HRPO secondary antibody (dilution 1:1000; AbD Serotec, UK) and the Dako EnVision HRP system (Carpinteria, CA, USA). Tissues were counterstained with methyl green.

Circulating tumour cells (CTC). Blood samples were collected from the lateral saphenous vein of the sham $(n=3)$ and pre-irradiated $(n=3)$ mice at 4 and 7 days after the injection of D2A1 FUCCI-expressing cells in the mammary glands. Samples diluted 1:10 in PBS were spread in a Petri dish. The presence of CTC in each blood sample was quantified by fluorescence microscopy from 10 images of representative areas (magnification $\times 100)$ that were acquired as described above.

Statistical analysis. Experimental data are presented as mean \pm s.e.m. Statistical analyses were performed using the nonparametric Mann-Whitney test. A $P$-value of $<0.05$ was considered significant.

\section{RESULTS}

Pre-irradiation of the mammary gland promotes the invasion and migration of mouse mammary cancer cells. To determine whether irradiation of the mammary gland provided a microenvironment conducive to the migration of cancer cells, we first investigated the effect of radiation on the invasion capacity of 
D2A1 cells in vitro by using invasion chambers. The BALB/c 3T3 fibroblasts were used to represent the stroma and were plated in the lower compartment of the chamber before being irradiated at 0 or $5 \mathrm{~Gy}$. Our results showed that irradiated fibroblasts acted as a chemoattractant, and increased by 1.7 -fold $(P=0.003)$ the number of D2A1 cells that crossed the Matrigel layer (Figure 2A).

Then, we assessed whether pre-irradiation of mice mammary gland had an effect on the migration of D2A1 FUCCI-expressing cells by using an animal optical imager. At 1 week after their injection close to the nipple, cells within the nonirradiated control mammary glands were forming a compact tumour at the site of implantation. In sharp contrast, in the pre-irradiated mammary glands, the D2A1 FUCCI-expressing cells had migrated away from the implantation site and were forming tumours adopting an elongated shape. The migration distance from the injection site to the front of the tumour was increased by 1.8 -fold $(P=0.0095)$ in the pre-irradiated mammary gland compared with the control nonirradiated one in the same animal (Figure $2 \mathrm{~B}$ and $\mathrm{C}$ ). Tumour volumes in the pre-irradiated mammary glands were also smaller (Figure 2D). This indicates that radiation favours the migration and invasion of cancer cells that occurred at the expense of tumour growth. The experiment was repeated in an independent group of mice, for whom none of the mammary glands had been irradiated. The distance of D2A1 FUCCI-expressing cell migration and growth within the mammary glands of these sham-treated mice was equivalent to those measured in the nonirradiated mammary glands of mice whose opposite mammary gland had been preirradiated. These results ruled out the possibility that systemic factors induced by radiation modified the migration of cancer cells implanted in the nonirradiated mammary gland. This supports the model of using a mouse in which one mammary gland is irradiated whereas the contralateral nonirradiated gland acts as a control, thus avoiding interanimal variations.

Effect of radiation on cell cycle distribution. Using the animal optical imager, only red fluorescence emitted by the D2A1 FUCCIexpressing cells was observed in both sides of the mammary gland. This suggested that either cancer cells were concentrated in the G1 phase or the green fluorescence was attenuated by tissues (Hillman et al, 2011). Therefore, histological analyses were performed on frozen tumour sections that revealed a high number of red and green cells. This result supports the hypothesis that green fluorescence was attenuated by tissues. The tumour sections were then used to assess the effect of pre-irradiation of the mammary
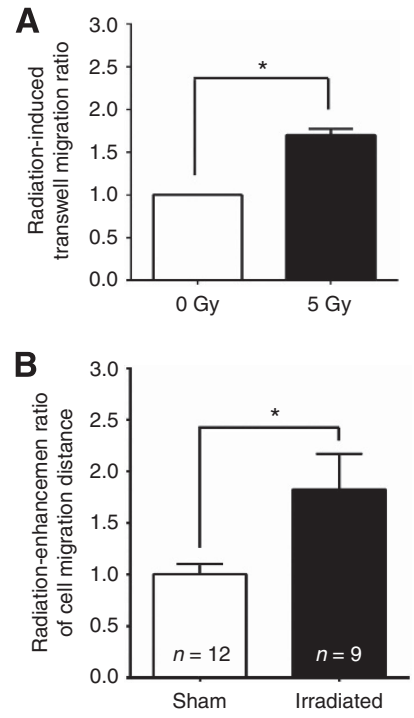

C
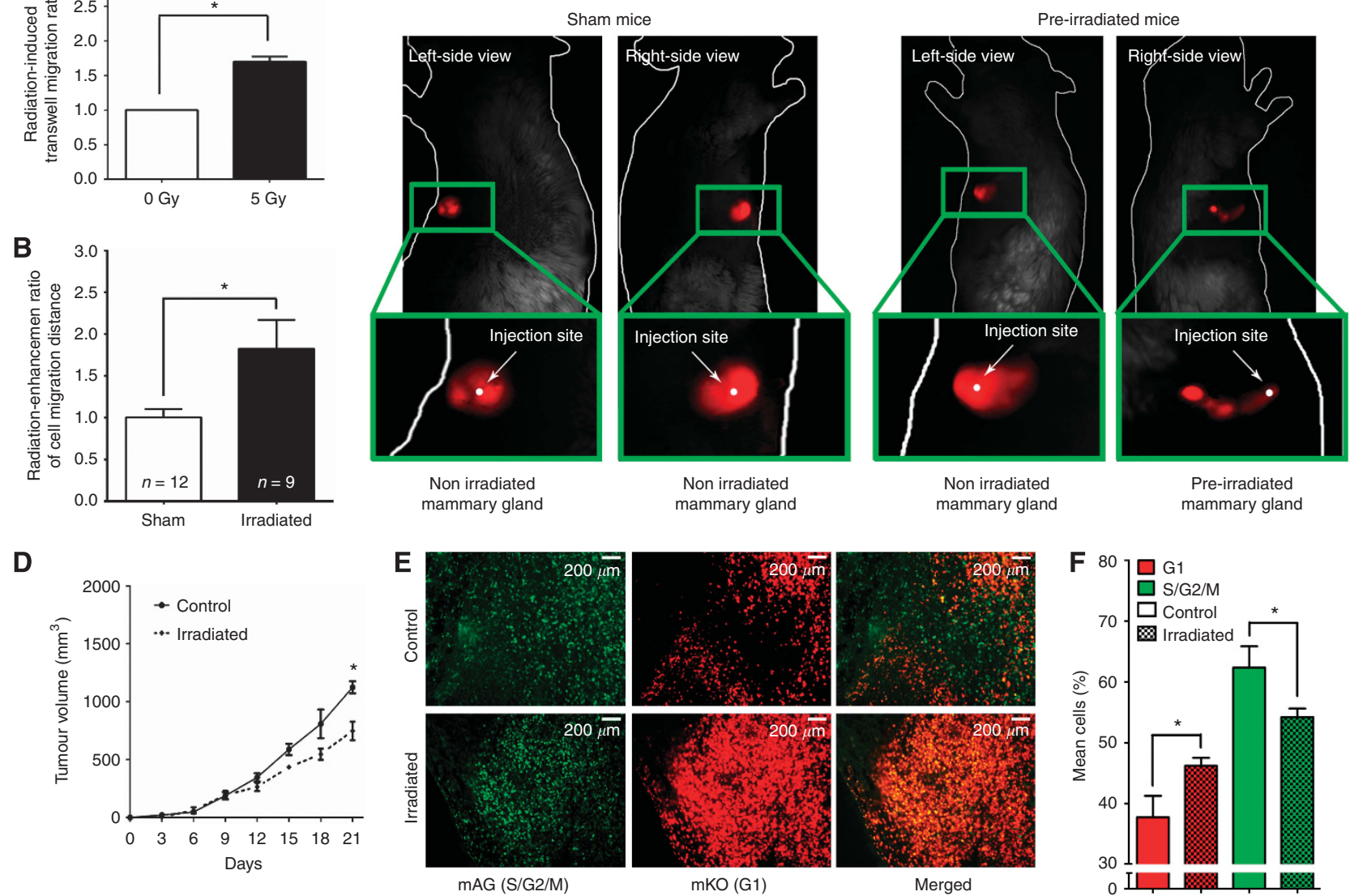

Figure 2. Migration of D2A1 FUCCl-expressing cancer cells. (A) In vitro transwell migration of D2A1 cancer cells. The D2A1 migration increased by 1.7-fold ( $P=0.003$ ) by irradiated ( $5 \mathrm{~Gy}$ ) BALB/c 3T3 plated in the bottom chamber. (B and $\mathbf{C})$ In vivo optical imaging of D2A1 FUCCl-expressing mouse mammary cancer cell migration within the mice mammary glands 1 week after being pre-irradiated or sham irradiated. Animals were irradiated on the right mammary gland and the left side was used as a nonirradiated control. Tumour cells were then implanted in both sides. For the sham-irradiated mice, which had not received any radiation dose, cancer cells were implanted in the mammary glands of both sides. No radiation-induced migration in the mammary glands was observed for the sham group $(n=12)$ compared with a 1.8-fold increase of cancer cell migration in pre-irradiated mice $(n=9 ; P=0.0095)$. Results were interpreted as radiation-enhancement ratios of cell migration in mammary glands. (D) Tumour volumes in pre-irradiated and nonirradiated mammary glands. (E) Representative fluorescence images of frozen sections of mammary tumours used to quantify cancer cells in S/G2/M (green) or G1 (red) phases. (F) Effect of radiation on cell cycle distribution. The mean increase of the ratio of red to green cells in pre-irradiated tissue was $26 \%(P=0.0356 ; n=12)\left({ }^{\star} P<0.05\right)$. 
gland on the proliferation of tumour cells by quantifying cells at the G1 phase (red fluorescence) and those in S/G2/M phases (green fluorescence). Radiation increased by $26 \%$ ratio of red-to-green cells $(P=0.0356)$ compared with the control tumours (Figure $2 \mathrm{E}$ and F). The correlation between the decrease of proliferating cells (green) and the stimulation of cancer cell migration supports that pre-irradiation of the mammary gland promotes the migration of cancer cells while reducing the proliferation rate of tumour cells.

Pre-irradiation of healthy mammary gland promotes lung metastases. To assess whether the stimulation of cancer cell migration induced in the pre-irradiated mammary gland affected the development of metastases, the number of lung metastases was quantified by optical imaging 21 days after the implantation of the D2A1 FUCCI-expressing cells. In the sham group, none of the mammary glands were irradiated before implantation of the D2A1 FUCCI-expressing cells on both sides. Although few metastases were observed in the lungs of sham-irradiated mice, the number of metastases in pre-irradiated animals increased by 2.4 -fold $(P=0.0281$; Figure $3 \mathrm{~A}$ and $\mathrm{B})$. Confirming the presence of metastases, frozen sections of lungs observed under fluorescence microscopy revealed strong red and green fluorescence signals emitted by the metastases (Figure 3C). The ratio of red-to-green cells in the lung metastases was not affected irrespective of whether the D2A1 cells were implanted in either the pre-irradiated or nonirradiated animals (results not shown). Supporting the hypothesis that irradiation of the mammary gland did not affect the proliferation rate of metastatic cells, the diameters of pulmonary metastases in irradiated and nonirradiated animals were not significantly different (Figure 3D). Pre-irradiation of the mammary gland increased the number of lung metastases but did not affect the metastatic cell proliferation rate.

Mechanisms involved in radiation enhancement of pulmonary metastases. We first hypothesised that the higher number of pulmonary metastases was caused by an increase of CTCs. The CTCs were easily distinguishable in blood samples and were quantified by fluorescence microscopy on days 4 and 7 after the implantation of the D2A1 FUCCI-expressing cells in the mammary glands. Mice subjected to mammary gland pre-irradiation showed a two-fold increase in CTC on days $4(P<0.0001)$ and 7 $(P=0.0001)$ (Figure 4A)
We next verified whether pre-irradiation of the mammary gland might have released systemic factors that would favour the extravasation of circulating cancer cells to the lungs. This was assessed by directly injecting the D2A1 FUCCI-expressing cells $\left(10^{6}\right)$ via the tail vein of mice with a pre-irradiated mammary gland, which were compared with a second group of nonirradiated mice. The animals were killed 9 days later and their lungs removed to quantify the metastatic area by optical imaging. The number of lung metastases was not significantly different between the sham and pre-irradiated groups, thus supporting the fact that the nesting of cancer cells in the lungs was not favoured by the pre-irradiation of a mammary gland (Figure 4B).

Assessment of promigratory and inflammatory factors. To characterise these adverse effects of radiation, promigratory and inflammatory factors were quantified in pre-irradiated mammary glands. As proteases are known to favour migration and invasion of cancer cells, the activity and/or levels of MMP-2 and MMP-9 were first determined by zymography. Surprisingly, no radiation enhancement was observed with both MMPs in the mammary glands that were either implanted with D2A1 tumour or free of D2A1 tumours (Figure 5A and B), as analysed by zymography. These results were validated by IHC analyses as heterogeneous increase of MMP-2/-9 could be missed when the analysis is done in the whole mammary glands by zymography. The IHC results confirmed that MMP-2 expression was not increased in irradiated and nonirradiated mammary glands free of D2A1 tumour (Figure 5CI and CII). Similar levels of MMP-2 (Figure 5C-III and C-IV) and MMP-9 (Figure 5CV and CVI) were also obtained in tumours implanted in irradiated or nonirradiated mammary glands. The MMP-2 was specifically localised in tumour periphery with almost no expression in the tumour core. The MMP-9 was moderately expressed everywhere in mammary tumours but homogeneously. Likewise, the expression of MT1-MMP, an activator of these proteases, was not stimulated by radiation (Figure 5D).

We then characterised several induced inflammatory molecules in irradiated mammary glands. The relative expression of IL- 6 was significantly increased $(P=0.0091)$, but not that of IL- $1 \beta$, TGF- $\beta 1$ or TNF- $\alpha$, as measured by $\mathrm{qPCR}$ at $6 \mathrm{~h}$ post irradiation. Regarding the pathway of biosynthesis of $\mathrm{PGE}_{2}$ and $\mathrm{PGD}_{2}$, a higher expression of COX-2 was found $(P=0.0039)$, whereas a modest
A

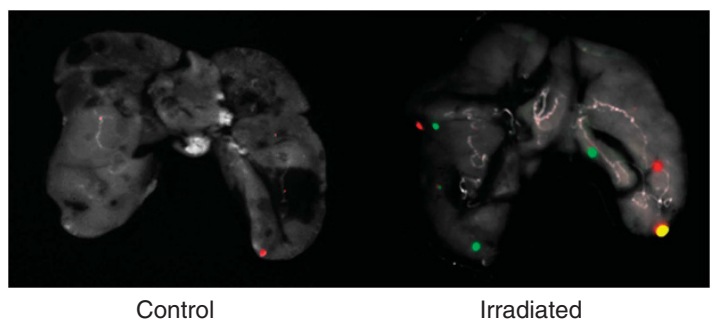

C

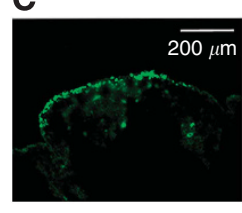

Green (mAG)

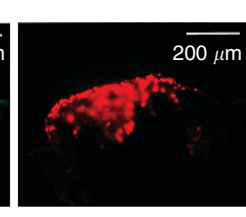

Red (mKO)

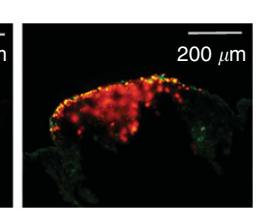

Merged
B
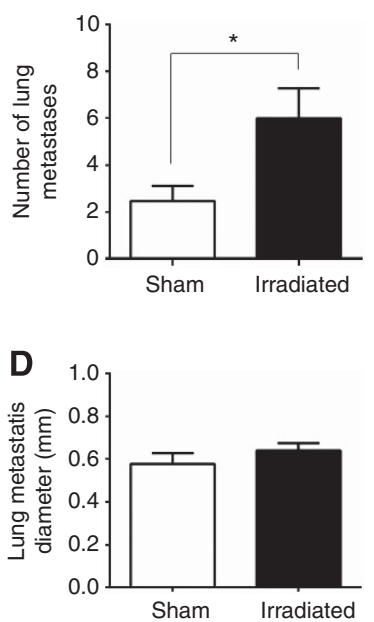

Figure 3. Pre-irradiation of the mammary gland increased the number of lung metastases. (A and B) In vivo optical imaging of lungs from irradiated and nonirradiated mice 21 days after cancer cells were implanted in the mammary gland. The number of lung metastases was 2.4 times greater in irradiated mice $\left(P=0.0281 ;{ }^{\star} P<0.05\right)$. (C) Fluorescence microscopy of a pulmonary metastasis. (D) Mean diameter of lung metastases from irradiated and sham mice. 

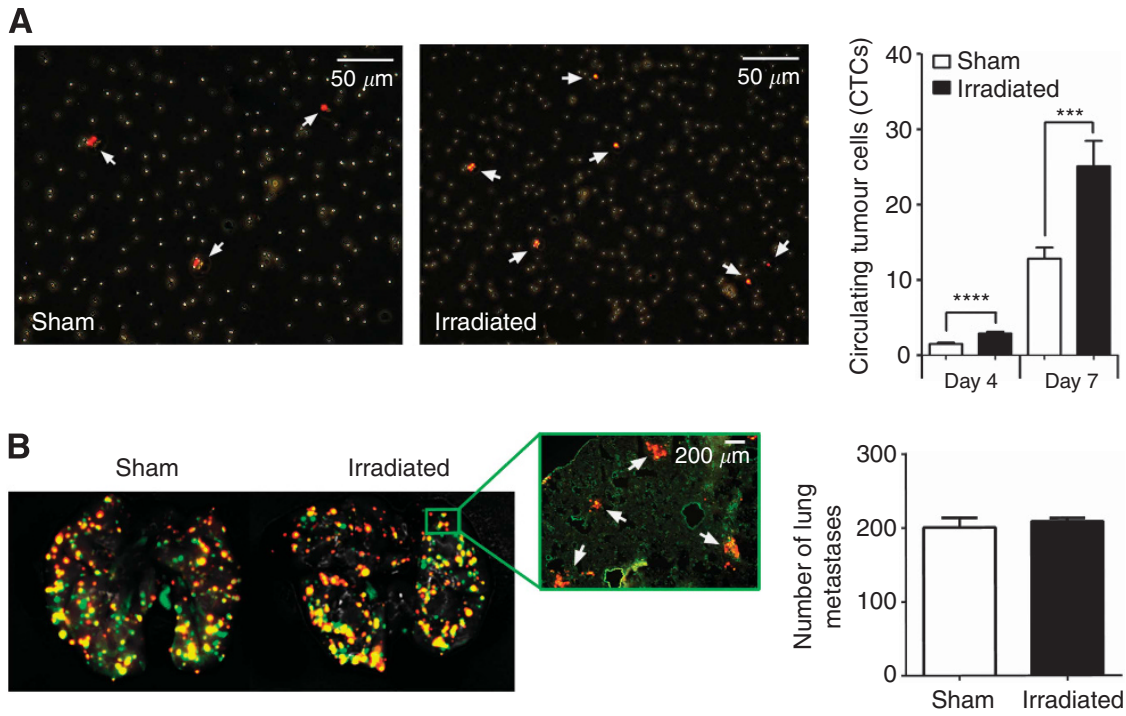

Figure 4. Mechanisms involved in radiation enhancement of pulmonary metastases. (A) Blood samples were collected at the indicated time after the implantation of DA21 FUCCl-expressing cancer cells in the mammary glands of pre-irradiated and sham-irradiated mice for CTC quantification. Images were acquired by fluorescence microscopy ( ${ }^{\star \star \star} P<0.05$; $\left.{ }^{* \star \star \star} P<0.05\right)$. (B) Optical imaging of pulmonary metastases 9 days after tail vein injection of D2A1 FUCCl-expressing cells. The presence of cancer cells in the lungs was confirmed by fluorescence microscopy (white arrows) and the number of lung metastases in frozen lung sections was quantified in representative areas.

but nonsignificant increase of PLA2 expression was also observed. Interestingly, 15-PGDH expression, which metabolises $\mathrm{PGE}_{2}$, was reduced (Figure 5D). The levels of prostaglandins $\mathrm{PGE}_{2}$ and $\mathrm{PGD}_{2}$, at different times post irradiation, were quantified by LC-MS/MS. A small increase, only for $\mathrm{PGE}_{2}$, was observed at 4 and $7 \mathrm{~h}$ post irradiation (Figure 5E).

\section{DISCUSSION}

Primary breast tumours are frequently removed by conservative surgery. However, 39-63\% of patients display malignant microfoci scattered throughout their breast (Holland et al, 1985). Therefore, protocols of radiotherapy include the whole breast and frequently a portion of the chest to include the axillary and supraclavicular lymph nodes. Consequently, a large volume of healthy tissue receives a significant radiation dose causing inflammation (Rodemann and Blaese, 2007).

The importance of the microenvironment in tumour progression is becoming increasingly accepted (Goldberg and Schwertfeger, 2010). As inflammation can be associated with the promotion of metastases, it is important to determine whether radiation-induced inflammation in healthy breast tissue could stimulate the migration of cancer cells and ultimately favour the formation of metastases.

An enhancement of cancer cell invasion after irradiation has been reported for pancreatic cancer cells (Qian et al, 2002), glioma cells (Wild-Bode et al, 2001; Park et al, 2006), melanoma cells (Rofstad et al, 2004; Kaliski et al, 2005), rectal carcinoma cells (Speake et al, 2005) and colon carcinoma cells (Wang et al, 2000). These studies were designed to measure the invasiveness of irradiated cancer cells that survived after radiation treatment. The present study was designed to investigate whether irradiation of the $\mathrm{BALB} / \mathrm{c}$ mouse mammary gland could stimulate the migration of mouse mammary cancer cells and the development of lung metastases. To test our hypothesis, mice mammary glands were pre-irradiated before implantation of the D2A1 mouse mammary cancer cells. This protocol eliminated confounding effects such as the selection of cancer cells more likely to migrate, which could occur by irradiating the tumour and the mammary gland at the same time.

Following irradiation of the mammary gland, a substantial stimulation of D2A1 cell migration occurred at the expense of the growth of the primary tumours, which were smaller and more elongated compared with the tumours implanted in nonirradiated mammary glands. A similar enhancement was measured in vitro using invasion chambers in which irradiated fibroblasts stimulated the invasiveness of nonirradiated D2A1 cells through a layer of Matrigel.

The radiation enhancement of cancer cell migration was a local effect limited to the pre-irradiated mammary gland. Indeed, migration of the D2A1 FUCCI-expressing cells in the opposite nonirradiated mammary gland was similar to the migration found in animals who did not have any of their mammary glands irradiated. Therefore, irradiation did not seem to release promigratory cytokines into the circulation that would favour the migration of cancer cells in nonirradiated tissues.

The ability of an irradiated tissue to favour migration of cancer cells at the expense of growth of the primary tumour was previously reported in a glioblastoma rat model (Desmarais et al, 2012). Brain irradiation before implantation of F98 glioma cells reduced the growth of the primary tumour and favoured the infiltration of cancer cells that migrated a longer distance from the edges of the primary tumour. Notably, this switch from a proliferation to infiltration phenotype of the F98 cells reduced the mean survival time of the animals.

The emergence of a migratory phenotype is believed to be the consequence of acquired mutations in cancer cells. However, in our model, stimulation of the migratory phenotype was observed without irradiating the cancer cells. These results led us to propose an alternative explanation based on pro-migratory molecules that trigger the transition from a proliferative phenotype to an invasive one. A mutation-based hypothesis alone cannot explain the metastatic progression of all tumours. For example, a mutationbased hypothesis fails to explain the short time to recurrence of glioblastoma multiforme (GBM) after tumour resection (Hatzikirou et al, 2010). Giese et al (2003) have reported that cell migration and proliferation are mutually exclusive processes for glioma cells. In their model, glioma cells proliferated only when 
A

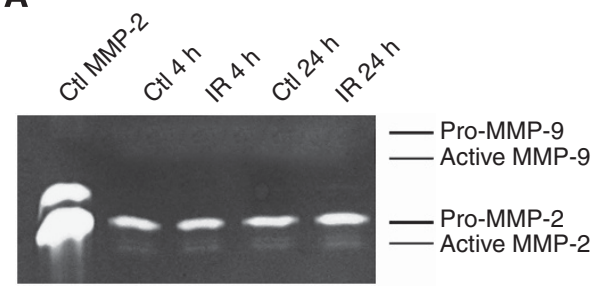

B

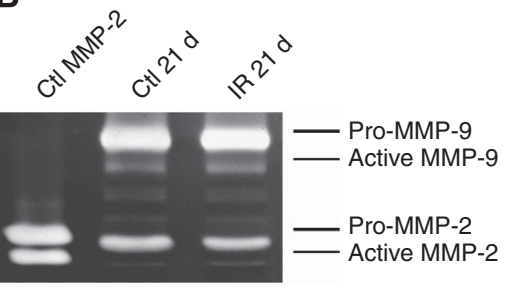

C
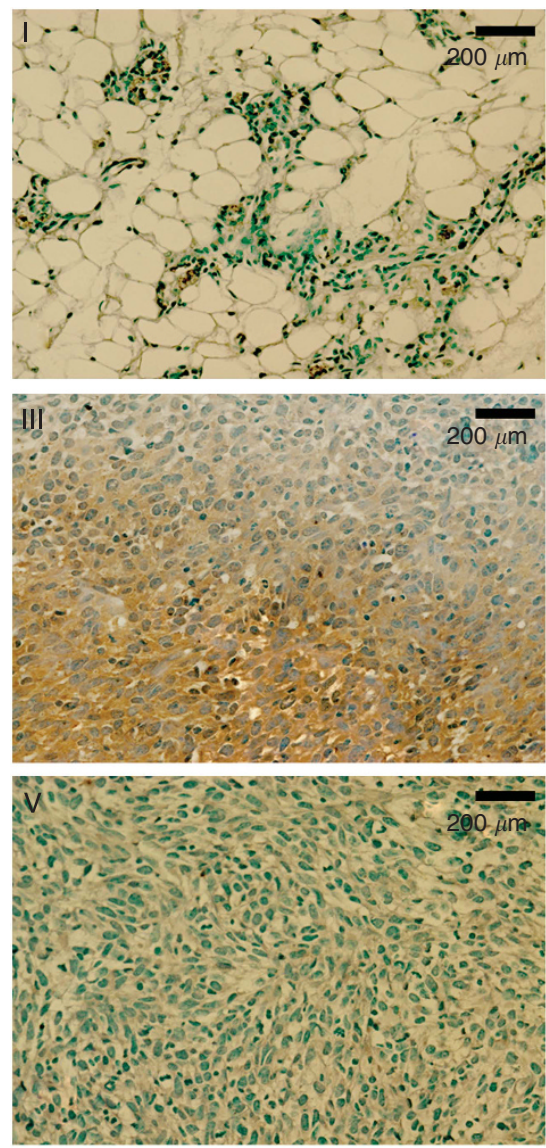

D

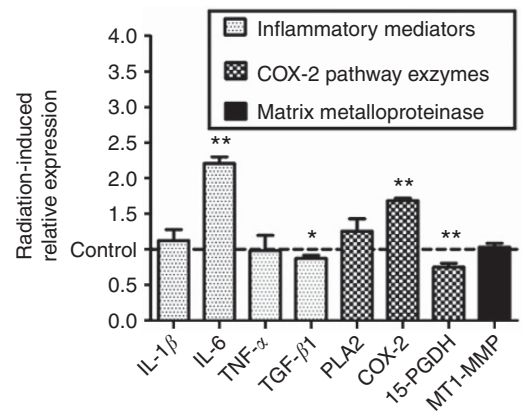

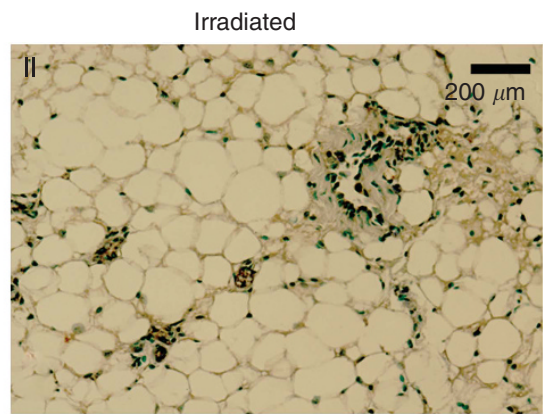
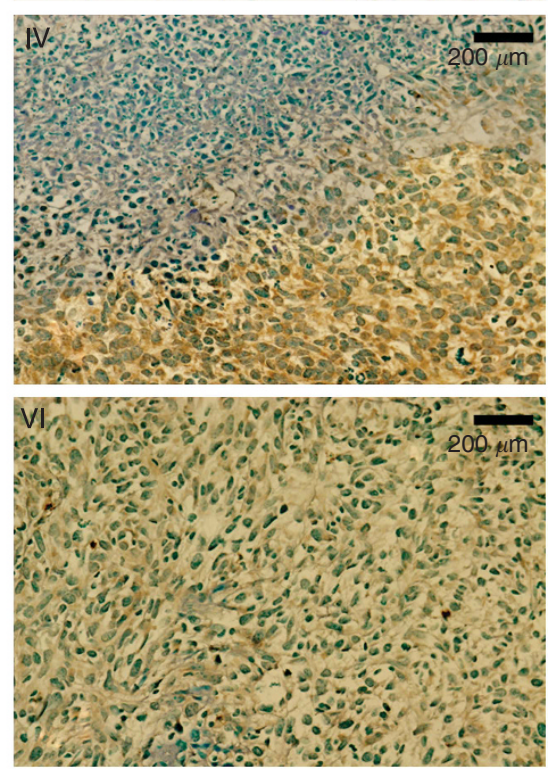

E

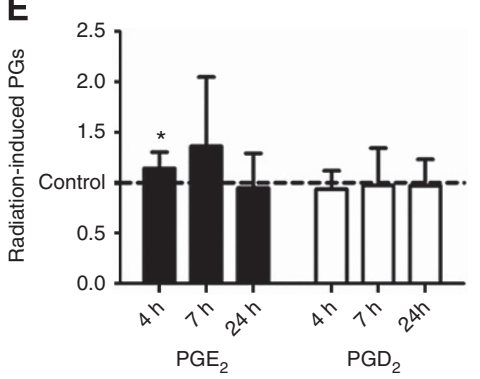

Figure 5. Radiation enhancement of pro-migratory and inflammatory factors. (A and B) Zymography analyses were performed on lysates of both irradiated and nonirradiated mammary glands that were, respectively, free of D2A1 cancer cells or bearing D2A1 cancer cells. Both MMP-2 and MMP-9 were detected in these analyses. (C) Immunohistochemistry of MMP-2 expression in control (I) and pre-irradiated mammary glands (II). Expression of MMP-2 (III and IV) and MMP-9 (V and VI) in D2A1 tumours implanted in pre-irradiated or not mammary glands. (D) The relative expression of genes potentially involved in cancer cell migration, as quantified by qPCR $6 \mathrm{~h}$ after the last session of irradiation. Relative mRNA expressions are plotted as a radiation enhancement ratio. (E) $P G E_{2}$ and $P G D_{2}$ were quantified by $L C-M S / M S$ at 4,7 and $24 \mathrm{~h}$ after the last session of irradiation. Results were plotted as radiation-induced PG ratios calculated with an internal standard $\left(P G E_{2} d^{4}\right)\left({ }^{\star} P<0.05 ;{ }^{\star \star} P<0.05\right)$.

they did not move. It turns out that the proliferation and migration of tumour cells are mutually exclusive phenotype. This mechanism, known as the migration/proliferation dichotomy (or the 'Go or
Grow' mechanism), is also supported by experimental evidence showing the lower proliferation rate of migratory cells in comparison with the tumour core (Giese et al, 2003). 
In our study with pre-irradiated mammary glands, we implanted D2A1 cells that expressed the FUCCI cell cycle marker. This tool allowed us to confirm a transition to the G1 phase and a depletion of the S/G2/M phases in the tumour cells implanted in pre-irradiated mammary glands, thus supporting a transition from the proliferative to migratory phenotypes.

Cell migration is a coordinated process, and it is likely that changes in the expression of several genes are required for cancer cells to become mobile. Carcinomas can undergo an epithelialto-mesenchymal transition (EMT) and then move through a matrix-filled space by using proteases (Nabeshima et al, 2002). Transforming growth factor- $\beta 1$ can increase the migration of cancer cells by inducing an EMT (Romagnoli et al, 2012). In the pre-irradiated mammary glands of BALB/c mice, TGF- $\beta 1$ gene expression was not increased. A similar result was reported by Barcellos-Hoff et al (1994) who described an increase of the activation of latent TGF- $\beta 1$ by radiation rather than an elevation of gene expression (Barcellos-Hoff, 1993). Moreover, inflammation is a very dynamic process. Our specific time point may have missed TGF- $\beta 1$ gene expression, as well as any other inflammatory mediators that were not reported to be increased by radiation in this study.

However, the majority of solid tumours do not undergo an EMT (Sahai, 2005). These cancer cells migrate by adopting an amoebid style of movement that does not require proteases because the cells are able to squeeze through gaps in the extracellular matrix (ECM) (Sahai, 2005). By using in vivo videomicroscopy, it was previously reported that D2A1 cells in mouse liver can squeeze through hepatocytes (Morris et al, 1994).

Levels of the MMP-2 and MMP-9 proteases were not significantly increased in the pre-irradiated mammary gland or in the D2A1 tumours. Nevertheless, MMP-2 was probably helping the migration of cancer cells as a high expression of this protease was found in the mammary glands. Supporting a potential role of MMP-2 in tumour progression, the IHC analyses demonstrated that MMP-2 was expressed exclusively in tumour periphery. We cannot also rule out the role for MMP-9 and the MMP activator MT1-MMP in radiation-induced migration because it was reported that radiotherapy increased by 2 - to 18 -fold the plasma level of MMP-9 in women with breast cancer (Riekki et al, 2000). We propose, in our animal model, that the increase of D2A1 cancer cell migration would be associated with amoeboid-like movement and MMP-2. Therefore, whether MMP inhibitors could have a beneficial role in the prevention of the radiation enhancement of metastasis remains to be assessed.

Extravasation of circulating cancer cells to organs is in part reliant on the expression of adhesion molecules like the intercellular adhesion molecule-1 (ICAM-1) and vascular cell adhesion molecule-1 (VCAM-1) on the surface of endothelial cells. Their expression can be stimulated by IL- $1 \beta$ and TNF- $\alpha$, but not by IL-6 (ten Kate et al, 2006). In our mouse model, only the expression of IL- 6 was promoted by radiation. The extravasation rate of circulating cancer cells did not seem to be affected by preirradiation of the mammary gland, as intravenous injection of D2A1 FUCCI-expressing cells in the tail vein led to a similar number of lung metastases in the pre-irradiated and nonirradiated animals. However, the stimulation of lung metastases induced by radiation was associated with an elevation of CTCs. The mechanisms responsible for this enhancement of CTC induced by pre-irradiation of the mammary gland are unclear. The increase in CTC in our model does not seem to be attributed to a stimulation of angiogenesis within the mammary gland. Vascular endothelial growth factor (VEGFA) expression within the mammary gland, as assessed by qPCR (Supplementary Figure 1A), was not enhanced $6 \mathrm{~h}$ after irradiation. The number of blood vessels did not increase either, which were quantified by immunohistochemistry with the CD31 endothelial cell marker

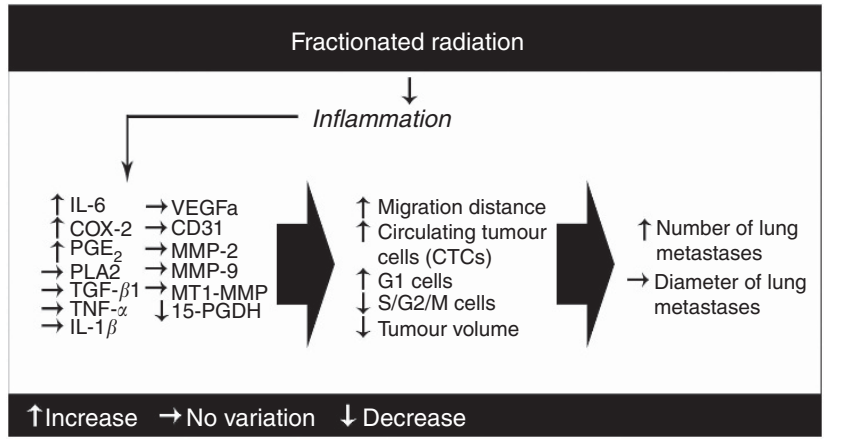

Figure 6. Model of breast cancer cell migration stimulated by radiation. We propose that inflammation mediated by fractionated radiation in breast cancer therapy causes a substantial enhancement of cell migration and CTC-promoting pulmonary metastases.

within the tumour-bearing pre-irradiated mammary gland (Supplementary Figure 1B and Supplementary Methods). However, whether pre-irradiation might, by increasing inflammatory cytokines, promote vascular permeability or damage to the basement membrane within the mammary gland (thereby facilitating access of the cancer cells to the circulation) will require further investigation.

Cyclooxygenase- 2 is a key enzyme in the inflammatory response that mainly produces $\mathrm{PGE}_{2}$. Notably, elevated expression of COX-2 in human breast cancer biopsies has been associated with distant metastases and poor prognoses (Ranger et al, 2004; Zerkowski et al, 2007). Although COX-2 is known to be upregulated by radiation (Yang et al, 2011), its inhibition was shown to decrease tumour growth, angiogenesis and metastasis in breast cancer mouse models (Chang et al, 2004; Greenhough et al, 2009; Tian and Schiemann, 2010). To counterbalance COX-2, $\mathrm{PGE}_{2}$ is degraded by 15-PGDH. Interestingly, Wolf et al (2006) showed that a low level of $15-\mathrm{PGDH}$ was found in highly metastatic breast carcinoma MDA-MB-231 cells and an upregulation of 15-PGDH significantly decreased their ability to form tumours in athymic mice. Our study supports such a role for COX-2 and $\mathrm{PGE}_{2}$ as a stimulation of $\mathrm{PGE}_{2}$ and a reduction of $15-\mathrm{PGDH}$ were concurrently associated with the promotion of cancer cell migration and lung metastases. We have also previously shown in vitro that $\mathrm{PGE}_{2}$ enhanced breast cancer cell invasion, whereas COX-2 inhibitor prevented radiation enhancement of breast cancer cell invasion (Paquette et al, 2011). Therefore, it would be interesting in future in vivo studies to evaluate whether the use of COX-2 inhibitors might represent an efficient way to prevent radiation-induced lung metastases.

In conclusion, we have shown in the current study that preirradiation of the mammary gland increased the migration of mouse mammary cancer cells, the quantity of circulating cancer cells and the number of lung metastases (Figure 6). These adverse effects were not due to mutations induced by radiation in cancer cells, but rather to pro-migratory molecules induced in the microenvironment of irradiated mammary glands. On the other hand, we cannot exclude that vascular and microenvironment changes occurring during tumour growth could also contribute to the migration of cancer cells after irradiation. In clinic, our results might suggest that the efficiency of radiotherapy could be improved by preventing the stimulation of cancer cell migration induced by radiation.

\section{ACKNOWLEDGEMENTS}

BP, RB and CS are members of the Fonds de la Recherche en Santé du Québec (FRSQ)-funded Centre de recherche clinique ÉtienneLeBel. CS is a FRSQ scholar and is also funded by a researcher of 
the Canadian Foundation for Innovation. We thank Dr Ann Chambers for generously providing the D2A1mouse mammary cancer cells. We thank Dr Asako Sakaue-Sawano for kindly providing the lentiviral vectors coding for the FUCCI genes. The medical physicists, Patrick Delage and Vincent-Hubert Tremblay, are thanked for their very helpful dosimetry calculations for mice irradiation. We also thank Dr Chang Shu Wang for the BED calculations for the animals. Finally, special thanks to Chantal Mitterer for all of the implementation techniques for the in vivo imaging. This research project was supported by the Canadian Institutes of Health Research (Grant 184671).

\section{CONFLICT OF INTEREST}

The authors declare no conflict of interest.

\section{REFERENCES}

Balin-Gauthier D, Delord JP, Rochaix P, Mallard V, Thomas F, Hennebelle I, Bugat R, Canal P, Allal C (2006) In vivo and in vitro antitumor activity of oxaliplatin in combination with cetuximab in human colorectal tumor cell lines expressing different level of EGFR. Cancer Chemother Pharmacol 57: 709-718.

Barcellos-Hoff MH (1993) Radiation-induced transforming growth factor beta and subsequent extracellular matrix reorganization in murine mammary gland. Cancer Res 53: 3880-3886.

Barcellos-Hoff MH (2010) Stromal mediation of radiation carcinogenesis. J Mammary Gland Biol Neoplasia 15: 381-387.

Barcellos-Hoff MH, Derynck R, Tsang ML, Weatherbee JA (1994) Transforming growth factor-beta activation in irradiated murine mammary gland. J Clin Invest 93: 892-899.

Barcellos-Hoff MH, Ravani SA (2000) Irradiated mammary gland stroma promotes the expression of tumorigenic potential by unirradiated epithelial cells. Cancer Res 60: 1254-1260.

Chang SH, Liu CH, Conway R, Han DK, Nithipatikom K, Trifan OC, Lane TF, Hla T (2004) Role of prostaglandin $\mathrm{E}_{2}$-dependent angiogenic switch in cyclooxygenase 2-induced breastcancer progression. Proc Natl Acad Sci USA 101: 591-591-596.

Clarke M, Collins R, Darby S, Davies C, Elphinstone P, Evans E, Godwin J, Gray R, Hicks C, James S, MacKinnon E, McGale P, McHugh T, Peto R, Taylor C, Wang Y. Early Breast Cancer Trialists' Collaborative Group (EBCTCG) (2005) Effects of radiotherapy and of differences in the extent of surgery for early breast cancer on local recurrence and 15-year survival: an overview of the randomised trials. Lancet 366: 2087-2106.

Desmarais G, Fortin D, Bujold R, Wagner R, Mathieu D, Paquette B (2012) Infiltration of glioma cells in brain parenchyma stimulated by radiation in the F98/Fischer rat model. Int J Radiat Biol 88(8): 565-574.

Gallet P, Phulpin B, Merlin JL, Leroux A, Bravetti P, Mecellem H, Tran N, Dolivet G (2011) Long-term alterations of cytokines and growth factors expression in irradiated tissues and relation with histological severity scoring. PLoS One 6: e29399.

Giese A, Bjerkvig R, Berens ME, Westphal M (2003) Cost of migration: invasion of malignant gliomas and implications for treatment. J Clin Oncol 21: $1624-1636$

Goldberg JE, Schwertfeger KL (2010) Proinflammatory cytokines in breast cancer: mechanisms of action and potential targets for therapeutics. Curr Drug Targets 11: 1133-1146.

Greenhough A, Smartt HJM, Moore AE, Roberts HR, Williams AC, Paraskeva C, Kaid A (2009) The COX-2/PGE 2 pathway: key roles in the hallmarks of cancer and adaptation to the tumour microenvironment. Carcinogenesis $\mathbf{3 0}$ $377-386$.

Hatzikirou H, Basanta D, Simon M, Schaller K, Deutsch A (2010) 'Go or Grow': the key to the emergence of invasion in tumour progression? Math Med Biol 29(1): 49-65.

Hillman EM, Amoozegar CB, Wang T, McCaslin AF, Bouchard MB, Mansfield J, Levenson RM (2011) In vivo optical imaging and dynamic contrast methods for biomedical research. Philos Transact A Math Phys Eng Sci 369: 4620-4643.
Holland R, Veling SH, Mravunac M, Hendriks JH (1985) Histologic multifocality of Tis, T1-2 breast carcinomas. Implications for clinical trials of breast-conserving surgery. Cancer 56: 979-990.

Kaliski A, Maggiorella L, Cengel KA, Mathe D, Rouffiac V, Opolon P, Lassau N, Bourhis J, Deutsch E (2005) Angiogenesis and tumor growth inhibition by a matrix metalloproteinase inhibitor targeting radiation-induced invasion. Mol Cancer Ther 4: 1717-1728.

Lemay R, Archambault M, Tremblay L, Bujold R, Lepage M, Paquette B (2011) Irradiation of normal mouse tissue increases the invasiveness of mammary cancer cells. Int J Radiat Biol 87: 472-482.

Madani I, De Neve W, Mareel M (2008) Does ionizing radiation stimulate cancer invasion and metastasis? Bull Cancer 95: 292-300.

Mantovani A, Allavena P, Sica A, Balkwill F (2008) Cancer-related inflammation. Nature 454: 436-444.

Morris VL, Koop S, MacDonald IC, Schmidt EE, Grattan M, Percy D, Chambers AF, Groom AC (1994) Mammary carcinoma cell lines of high and low metastatic potential differ not in extravasation but in subsequent migration and growth. Clin Exp Metastasis 12: 357-367.

Nabeshima K, Inoue T, Shimao Y, Sameshima T (2002) Matrix metalloproteinases in tumor invasion: role for cell migration. Pathol Int 52: 255-264.

Paquette B, Therriault H, Desmarais G, Wagner R, Royer R, Bujold R (2011) Radiation-enhancement of MDA-MB-231 breast cancer cell invasion prevented by a cyclooxygenase-2 inhibitor. Br J Cancer 105: 534-541.

Park CM, Park MJ, Kwak HJ, Lee HC, Kim MS, Lee SH, Park IC, Rhee CH, Hong SI (2006) Ionizing radiation enhances matrix metalloproteinase-2 secretion and invasion of glioma cells through Src/epidermal growth factor receptor-mediated p38/Akt and phosphatidylinositol 3-kinase/Akt signaling pathways. Cancer Res 66: 8511-8519.

Qian LW, Mizumoto K, Urashima T, Nagai E, Maehara N, Sato N, Nakajima M, Tanaka M (2002) Radiation-induced increase in invasive potential of human pancreatic cancer cells and its blockade by a matrix metalloproteinase inhibitor, CGS27023. Clin Cancer Res 8: 1223-1227.

Rak JW, McEachern D, Miller FR (1992) Sequential alteration of peanut agglutinin binding-glycoprotein expression during progression of murine mammary neoplasia. Br J Cancer 65: 641-648.

Ranger GS, Thomas V, Jewell A, Mokbel K (2004) Elevated cyclooxygenase-2 expression correlates with distant metastases in breast cancer. Anticancer Res 24: 2349-2351.

Riekki R, Jukkola A, Sassi ML, Hoyhtya M, Kallioinen M, Risteli J, Oikarinen A (2000) Modulation of skin collagen metabolism by irradiation: collagen synthesis is increased in irradiated human skin. Br J Dermatol 142: 874-880.

Rodemann HP, Blaese MA (2007) Responses of normal cells to ionizing radiation. Semin Radiat Oncol 17: 81-88.

Rofstad EK, Mathiesen B, Galappathi K (2004) Increased metastatic dissemination in human melanoma xenografts after subcurative radiation treatment: radiation-induced increase in fraction of hypoxic cells and hypoxia-induced up-regulation of urokinase-type plasminogen activator receptor. Cancer Res 64: 13-18.

Romagnoli M, Belguise K, Yu Z, Wang X, Landesman-Bollag E, Seldin DC, Chalbos D, Barille-Nion S, Jezequel P, Seldin ML, Sonenshein GE (2012) Epithelial-to-mesenchymal transition induced by TGF-betal is mediated by Blimp-1-dependent repression of BMP-5. Cancer Res 72: 6268-6278.

Sahai E (2005) Mechanisms of cancer cell invasion. Curr Opin Genet Dev 15 $87-96$.

Sakaue-Sawano A, Kurokawa H, Morimura T, Hanyu A, Hama H, Osawa H, Kashiwagi S, Fukami K, Miyata T, Miyoshi H, Imamura T, Ogawa M, Masai H, Miyawaki A (2008) Visualizing spatiotemporal dynamics of multicellular cell-cycle progression. Cell 132: 487-498.

Speake WJ, Dean RA, Kumar A, Morris TM, Scholefield JH, Watson SA (2005) Radiation induced MMP expression from rectal cancer is short lived but contributes to in vitro invasion. Eur J Surg Oncol 31: 869-874.

ten Kate M, Hofland LJ, van Koetsveld PM, Jeekel J, van Eijck CH (2006) Pro-inflammatory cytokines affect pancreatic carcinoma cell. Endothelial cell interactions. JOP 7: 454-464.

Tian M, Schiemann WP (2010) PGE2 receptor EP2 mediates the antagonistic effect of COX-2 on TGF-beta signaling during mammary tumorigenesis. FASEB J 24: 1105-1116.

Wang JL, Sun Y, Wu S (2000) Gamma-irradiation induces matrix metalloproteinase II expression in a p53-dependent manner. Mol Carcinog 27: 252-258.

Wild-Bode C, Weller M, Wick W (2001) Molecular determinants of glioma cell migration and invasion. J Neurosurg 94: 978-984. 
Wolf I, O’Kelly J, Rubinek T, Tong M, Nguyen A, Lin BT, Tai HH, Karlan BY, Koeffler HP (2006) 15-Hydroxyprostaglandin dehydrogenase is a tumor suppressor of human breast cancer. Cancer Res 66: 7818-7823.

Wu P, van Overbeek M, Rooney S, de Lange T (2010) Apollo contributes to G overhang maintenance and protects leading-end telomeres. Mol Cell 39: 606-617.

Yang HJ, Youn H, Seong KM, Yun YJ, Kim W, Kim YH, Lee JY, Kim CS, Jin YW, Youn B (2011) Psoralidin, a dual inhibitor of COX-2 and 5-LOX, regulates ionizing radiation (IR)-induced pulmonary inflammation. Biochem Pharmacol 82: 524-534.

Yang P, Felix E, Madden T, Fischer SM, Newman RA (2002) Quantitative high-performance liquid chromatography/electrospray ionization tandem mass spectrometric analysis of 2- and 3-series prostaglandins in cultured tumor cells. Anal Biochem 308: 168-177.

Zerkowski MP, Camp RL, Burtness BA, Rimm DL, Chung GG (2007)

Quantitative analysis of breast cancer tissue microarrays shows high cox-2 expression is associated with poor outcome. Cancer Invest 25: 19-26.

This work is published under the standard license to publish agreement. After 12 months the work will become freely available and the license terms will switch to a Creative Commons AttributionNonCommercial-Share Alike 3.0 Unported License.

Supplementary Information accompanies this paper on British Journal of Cancer website (http://www.nature.com/bjc) 